Review

\title{
High Throughput Multiplex Serological Testing in Microbiology
}

\author{
Jonas Blomberg * \\ Department of Medical Sciences, Uppsala University; \\ * Correspondence: Jonas.blomberg@medsci.uu.se; Tel.: +46761131 231
}

\begin{abstract}
High throughput multiplex serological systems enable the small developer to set up tests at small cost, for microbes for which there are no commercial tests, and for aspects which have not been addressed by them. An example is testing for Zika and Tick Borne Encephalitis virus antibodies, where antigenic cross-reactions make diagnosis problematic. Our technique variant, Suspension Multiplex Immunoassay (SMIA) allows many samples to be tested for antibodies to many antigens in a short time. Computational compensation for cross-reactions is possible if a SMIA panel contains most of the potentially cross-reacting antigens. Using antibody avidity and pattern of reactivity to whole virus and nonstructural protein, antibodies due to vaccination and infection, respectively, as well as probable degree of protection, can be determined with high throughput. These multiplex techniques hold great promise for future diagnostic development. Theoretically, even large scale serological monitoring, like blood donor pathogen testing, could be done inexpensively and rationally with multiplex serology developed in house. However, the quality control demands are steep and in most cases out of scope for a single laboratory. There remain however a number of clinical applications where in house multiplex serology can be performed with adequate quality control under high throughput conditions.
\end{abstract}

Keywords: Multiplex serology, serosurveillance, vaccine monitoring, emerging diseases, clinical microbiology

\section{Introduction}

Let us not forget that it is the clinical need, not the commercial diagnostic company, which should determine which tests that we use. The promise of high throughput multiplex testing, to have all, at once, sounds too good to be true. But technological advances have made it possible. There are no particular reasons why high throughput multiplex testing should be inferior to conventional singleplex immunoassays. I here review the possibilities and remaining obstacles. Some applications are given in Table 1.

Broadly targeted syndromic panels have now entered clinical microbiological routine at the nucleic acid side. However, broad serological panels are also feasible and are useful for large scale seroepidemiology and surveillance of incidence and vaccine coverage.

The validation of a serological assay requires a collection of true positives and true negatives, and serial sera from clinical cases. At least one established "gold standard" test must be run alongside the new serological assay. Establishment of serological tests for newly discovered viruses, for which there are no established tests, requires careful collection of sera from clinical cases diagnosed by nucleic acid or other tests. It is through a recursive effort using a patchwork of gradually accumulated evidence to establish that the new serological method actually measures what it is intended to measure. It can be laborious for a singleplex assay. In a 25 plex assay, a 25 fold higher effort is required. This is one explanation for the slow emergence of multiplex serological 
assays. There is also more money to be earned by running several singleplex assays in parallel than by running one multiplex panel.

\section{Experience from previous development of multiplex serologies.}

Are there no shortcuts to accelerate to uptake of this rational technology? One trick is to have a collection of sera from 0.5-2 year-old children. Such a collection can be counted on being rich in "true negative" sera, good for establishing cutoffs. Another trick is to include "sleeper" antigens in a broadly targeted syndromic serological panel. The "sleeper" antigens are still not well characterized. They are there there simply as sentinels. A reaction may be true or false, but serves to alert that antibodies to the microbe may be present. The sample and the case can then be investigated using other techniques. "Sleeper" antigens must be chosen with caution. For example, testing with a "sleeper" HIV antigen without consent from the patient would probably be deemed unethical.

Including several antigens per microbe (like in a Western Blot) allows the likelihood of a true serological reaction to be raised if there is a reaction to several antigens from the same microbe. A microbe carries a broad collection of antigens to which antibodies are elicited concomitantly during an infection. This strategy is indispensable when new or rare microbes are tested for. But it is also helpful for distinguishing different kinds of antibody response to a microbe, like distinction of vaccine induced antibodies from antibodies arising from an infection. This is the subject of a patent which we applied for (Figure 1).

In my group, we have developed a multiplex serological technique called Suspension Multiplex Immunoassay (SMIA) (Figure 2) [1-16]. We have used it for research on diagnosis and seroprevalence. The SMIA technique is inexpensive and rational. It shows that the price of high throughput multiplex testing does not need to be high. It can be run with highly automated equipment, justifying the term high throughput. A comparable collection of singleplex ELISAs, if it exists, will nearly always be more expensive and laborious. SMIA consumes less of expensive antigen, and less sample, than standard singleplex assays [2]. The ability to economize with antigen enables use of purified, more costly, antigens. At serum dilutions of 1/100 and higher, the SMIA signal is proportional to amount of antibody. A microliter of serum can then give results from many (say, 50) antigens. So far, sensitivity has been similar to that of comparable commercial tests [2-4]. The SMIA result is in the form of MFI, Median Fluorescent Intensity. The signal is the median of many determinations, often 100 per antigen. This gives a high precision. If differences in reactivity are studied within the same reaction volume (generally a microtiter plate well), the accuracy of the intrawell comparison becomes very high. The main source of interwell variation is the pipetting error.

SMIA is also a good tool for infectious disease research, allowing rare and less well known microbe antigens to be included as "sleepers" with marginally increased cost. As experience increases, the "sleepers" may be included as regular and validated members of the serological panel. The recent large expansion of knowledge regarding microbes, both viruses, bacteria, fungi and protozoa brought by next generation sequencing necessitates an expansion of syndromic serological panels. The hundreds of newly discovered microbes are often only known by their sequence. We have found long synthetic peptides derived directly from sequence to be quick and rational alternatives 
when setting up initial serological panels for these hitherto unknown microbes. Further method optimization will reveal how much of synthetic peptides, recombinant proteins or whole microbe is needed.

\subsection{Computational compensation for serological cross-reactions}

A special aspect of multiplex serology is the ability to compensate for serological cross-reactions between similar microbes. Serology in the Flavivirus family, which includes Dengue, Zika, Yellow fever, Japanese encephalitis virus, Tick-borne encephalitis virus and Usutu, is notoriously difficult, due to frequent antigenic cross-reactions. An overview of the cross reactions we encountered in given in Figure 3. Seeing the reactivities of many antigens in a multiplex test (looking through a panorama window) instead of looking at single antigen reactions (looking through a key hole) allows distinction of "true" from "false" reactions in many cases.

\subsection{Use of antibody avidity for distinguishing serological cross-reactions from true reactions and potentially predict protection}

We also found that cross-reactions (false positive) frequently have lower avidity than true positive reactions. We devised a computational system which takes both cross-reactivity pattern with IgM and IgG, avidity and temporal pattern of evolution between several samples from the same patient into account. The system was able to promptly and correctly distinguish 47 Dengue from 13 Zika infection [3], and 5 Zika cases in over 100 returning travelers (unpublished).

The distinction of antibodies due to infection from those due to vaccination is especially problematic in countries with frequent TBEV vaccination and frequent TBEV exposure, like in Sweden. Our SMIA technique proved to be useful for separate measurement of seroprevalence of TBEV infection and vaccination (Figure 4). The principle could be extended to surveillance of coverage for all inactivated virus vaccines where there is a nonstructural antigenic virus protein which is not present in the vaccine, like Japanese encephalitis and polio viruses. It was also possible to use IgG avidity in SMIA for prediction of TBEV neutralization titer (Figure 5). This may enable high throughput surveillance of vaccine coverage in whole populations, and for individuals who want to know their TBEV protection status. The simplicity of SMIA avidity testing contrasts with the necessity of running neutralization tests under biosafety level 3, involving handling of the pathogenic TBEV.

\section{Possible developments}

Large scale vaccine serosurveillance may be an especially useful application for multiplex serology. The recent emergence of vaccine hesitancy [17] and resulting epidemics of virus diseases like measles [18] and mumps [19] requires an improved strategy for ensuring herd immunity. Multiplex serology using vaccine antigens can give the ability to quickly and inexpensively deliver a broad estimation of vaccine coverage to both individuals and population. It is reasonable to ask for vaccine immunity in potentially exposed personnel. But also children and their parents may get a concrete impetus to vaccination if the test shows absence of antibodies in a child. This may be more acceptable to vaccine sceptics rather than just trying to implement general vaccination. It is our experience that vaccine antigens can be directly coupled to SMIA beads. Thus, a general vaccine 
panel including measles, mumps, rubella, polio, hepatitis a, hepatitis b, hpv and rotavirus vaccine antigens could probably be created with low cost and effort.

\subsection{Limitations for in house multiplex serologies}

Is multiplex serology, like SMIA, ready to take on all serological testing? No. As mentioned in the beginning, every component in a multiplex test must be fully validated. For example, the optimization of blood donor singleplex testing for HIV, HTLV, Hepatitis B and C, has taken several decades. Not only have the serological methods been optimized for sensitivity and specificity, there have also been great advances in automation and technology for the tests. This testing is officially regulated with high demands on accuracy and quality control. To better this with an in house multiplex technology like SMIA is a major undertaking, out of scope for the single developer. For example, HIV antibody testing requires access to seroconversion and rare virus variant sera. The big diagnostic companies have had to buy these scarce resources for large sums.

\subsection{Some desirable clinical uses of multiplex serology}

There remain however a number of clinical applications where multiplex serology can be performed with adequate quality control under high throughput conditions (Table 2). Every clinical microbiologist knows that nucleic acid testing often must be complemented with serology to reach a probable diagnosis. Panels under construction include diagnosis of exanthema, jaundice, meningoencephalitis, immunosuppression, pregnancy infections and several zoonosis panels.

\section{Conclusions}

The reasonable costs and rationality of multiplex serologies like SMIA gives the small developer an opportunity to break new diagnostic ground in microbiology. It is fun to play with this tool.

\section{Tables}

Table 1. Some situations in which high throughput multiplex serological testing can be applied.

$\begin{array}{ll}\text { Research } & \text { public health } \\ \text { Serosurveys } & \text { Blood bank testing } \\ \text { Microbe discovery } & \text { Serological diagnostics } \\ \text { Epitope discovery } & \text { Vaccine monitoring } \\ & \text { Pathogen monitoring }\end{array}$

\section{Clinical routine and}

\section{public health}

Pathogen monitoring 
Table 2. Our ongoing work with syndromic and virus family oriented clinical SMIA panels. All are used for both IgG and IgM serology. Tested=Antigens tested. Publ=Publication. Abbreviations of zoonotic viruses are according to $[20,21]$.

\begin{tabular}{|c|c|c|c|}
\hline Panel & Diagnostic target & Major taxon & Antigens \\
\hline \multirow[t]{7}{*}{ Exanthema viruses } & Diagnosis of exanthema & Herpesviridae & HHV6b, (HHV6a) \\
\hline & & & HHV7 \\
\hline & & & VZV \\
\hline & & Parvoviridae & Parvovirus B19V \\
\hline & & Paramyxoviridae & Measles virus \\
\hline & & Togaviridae & Rubella virus \\
\hline & & Picornaviridae & Enterovirus general \\
\hline \multirow[t]{8}{*}{ Hepatitis viruses } & Diagnosis of jaundice & Picornaviridae & Hepatis A virus \\
\hline & & Hepadnaviridae & Hepatitis B virus \\
\hline & & Flaviviridae & Hepatitis $C$ virus \\
\hline & & Deltavirus & Hepatitis D virus \\
\hline & & Hepeviridae & Hepatitis E virus \\
\hline & & Herpesviridae & Cytomegalovirus \\
\hline & & & Epstein Barr virus VCA, EBNA1 \\
\hline & & Adenoviridae & Adenovirus hexon \\
\hline \multirow[t]{17}{*}{ Neurotropic viruses } & Diagnosis of meningoencephalitis & Paramyxoviridae & Mumps virus \\
\hline & & & Measles virus \\
\hline & & Picornaviridae & Polio virus \\
\hline & & & Enterovirus general \\
\hline & & & Parechovirus, e.g. type 1 and 3 \\
\hline & & Flaviviridae & TBEV, structural and nonstructur \\
\hline & & & Japanese encephalitis virus \\
\hline & & & West Nile virus \\
\hline & & Polyomaviridae & JC virus \\
\hline & & Herpesviridae & Herpes simplex type 1 \\
\hline & & & Herpes simplex type 2 crossreacti \\
\hline & & & Herpes simplex type 2 type specif \\
\hline & & & VZV \\
\hline & & & CMV \\
\hline & & & EBV VCA, EBNA1 \\
\hline & & & HHV6a and HHV6b \\
\hline & & & HHV7 \\
\hline \multirow[t]{3}{*}{ "TORCH" agents } & Pathogen serology during pregnancy & Protozoa & Toxoplasma gondii \\
\hline & & Parvoviridae & Parvovirus B19V \\
\hline & & Herpesviridae & CMV \\
\hline
\end{tabular}


6 of 13

\begin{tabular}{|c|c|c|c|}
\hline & & Togaviridae & Rubella \\
\hline \multirow[t]{16}{*}{ Immunosuppression } & $\begin{array}{l}\text { Pathogens active in immunosuppr. } \\
\text { patients }\end{array}$ & Protozoa & Toxoplasma gondii \\
\hline & & Polyomaviridae & JC virus \\
\hline & & & BK virus \\
\hline & & & Other polyomaviruses \\
\hline & & Adenoviridae & Adenovirus hexon \\
\hline & & Herpesviridae & Herpes simplex type 1 \\
\hline & & & Herpes simplex type 2 \\
\hline & & & VZV \\
\hline & & & EBV VCA, EBNA1 \\
\hline & & & HHV6a, HHV6b \\
\hline & & & HHV7 \\
\hline & & Parvoviridae & Parvovirus B19V \\
\hline & & & Other parvoviruses \\
\hline & & Anelloviridae & Anellovirus TTV1 \\
\hline & & & Other Anelloviruses \\
\hline & & Flaviviridae & Pegivirus (GBV-C) \\
\hline \multirow[t]{5}{*}{ Zoonosis panels } & Flavivirus infection & Flaviviridae & $\begin{array}{l}\text { TBEV, DENV, YFV, ZIKV, WN } \\
\text { USUV }\end{array}$ \\
\hline & Hantavirus infection & Hantavirus & PUUV, SNNV, KHFV, ANDV, DC \\
\hline & Filovirus infection & Filoviridae & ZEBOV, BEBOV, RESTV, MARV \\
\hline & Other Viral Zoonoses & & Lassa, CCHFV, RVFV, CHIKV, O \\
\hline & & & Rabies, SARS, MERS \\
\hline
\end{tabular}




\section{Original MFI - cutoff}

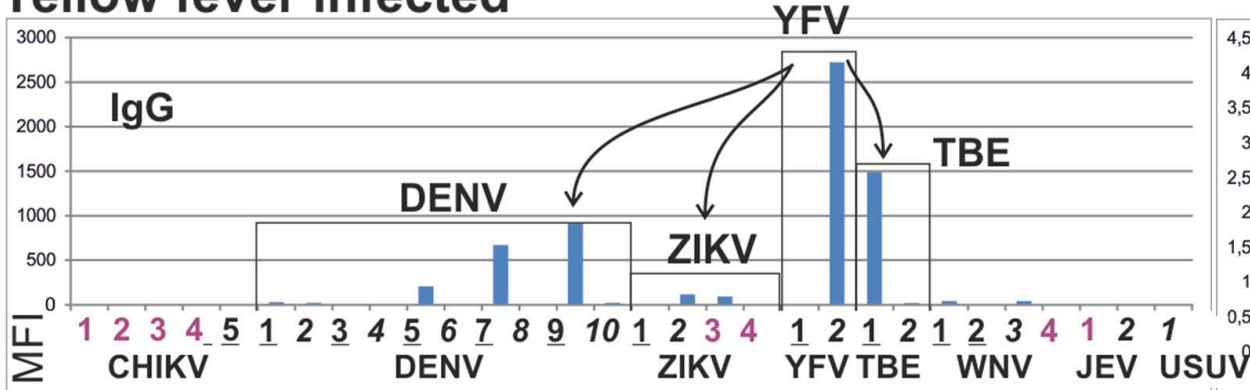

\section{West Nile virus infected}

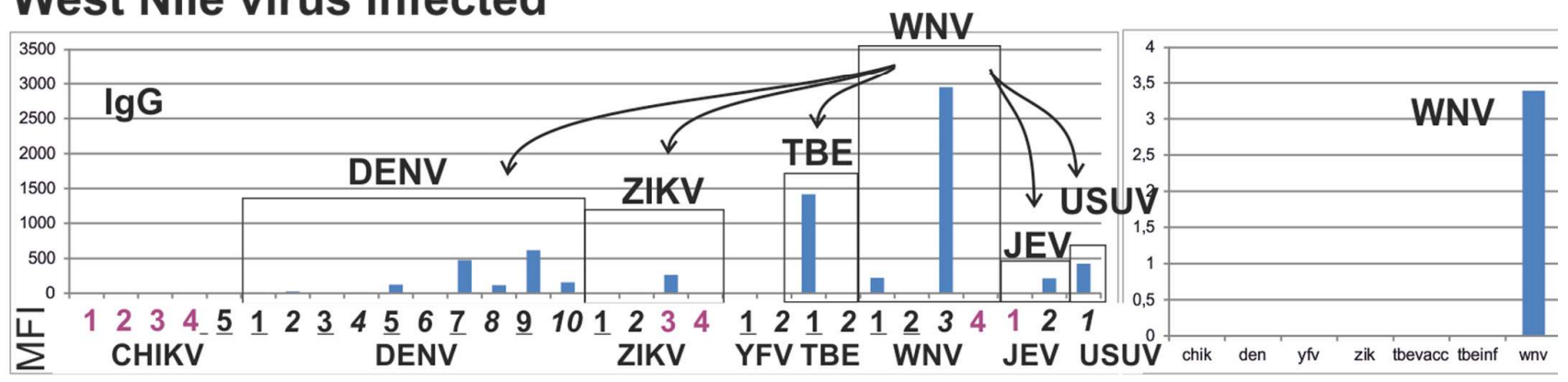

Figure 1. Principle behind computational compensation for antigenic cross reactions in a multiplex serology. Upper panel (A): Multiplex flavivirus serology with a serum from a yellow fever virus (YFV) infected individual. Cross reactions from YFV to other flaviviral antigens are shown. Lower panel (B). Cross-reactions occurring in a case of West Nile virus (WNV) infection. Applying a compensatory algorithm ("data reduction") disambiguates cross-reactions and suggests the infecting pathogen. Virus abbreviations are as in [20,21]. Flaviviral antigens are numbered from 1 up to 10. Underscored numbers: whole virus antigen. Red numbers: recombinant envelope proteins. Italicized numbers: recombinant NS1 protein. The method is further described in [3,24]. 


\section{SMIA w avidity}

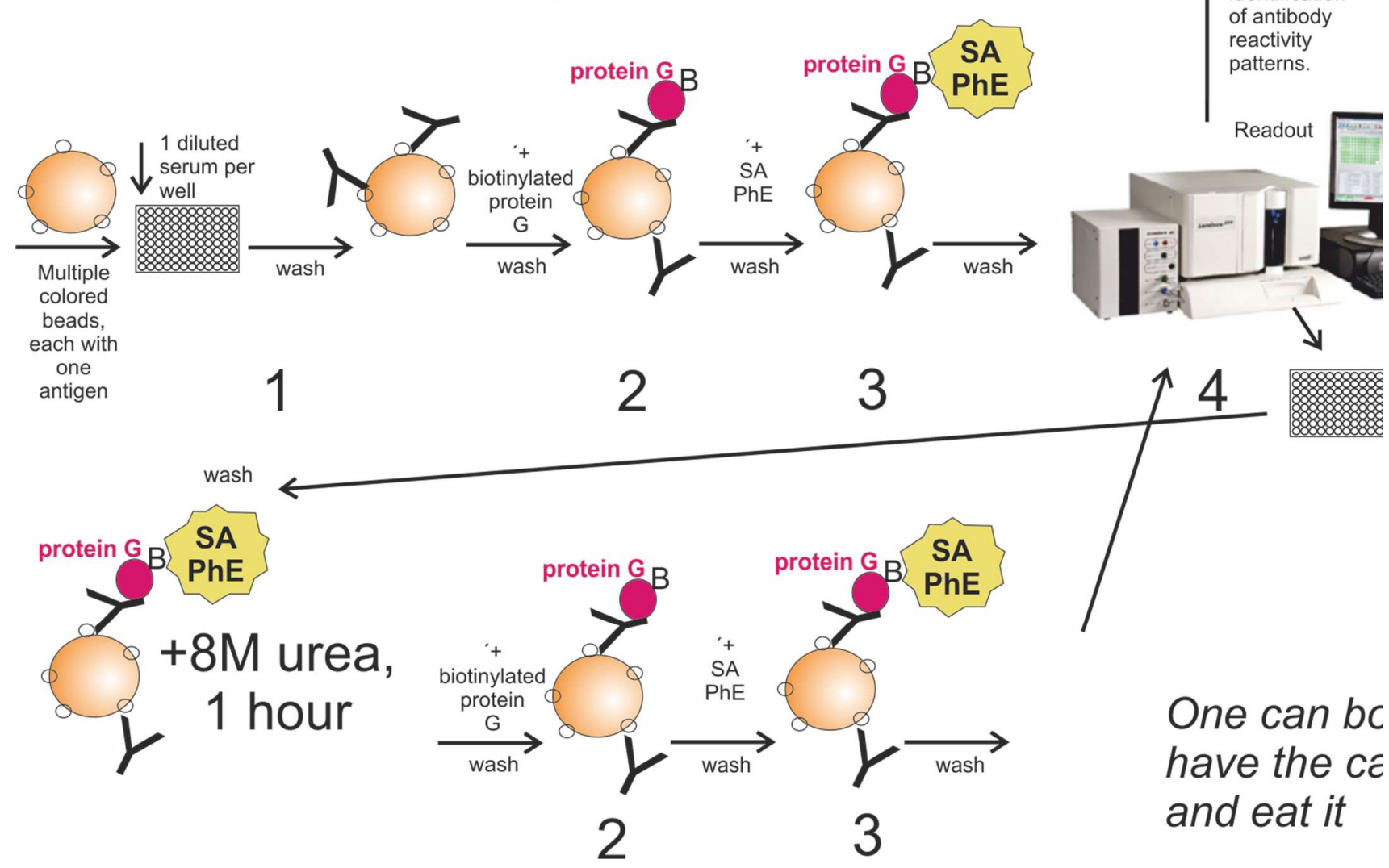

Figure 2. Principle behind SMIA, direct bioinformatic interpretation and avidity determination. The system can handle thousands of sera per day, and, depending on the antigen panel, measure and interpret over 100 antibody specificities in each serum. The principle is described in [24]. 
9 of 13

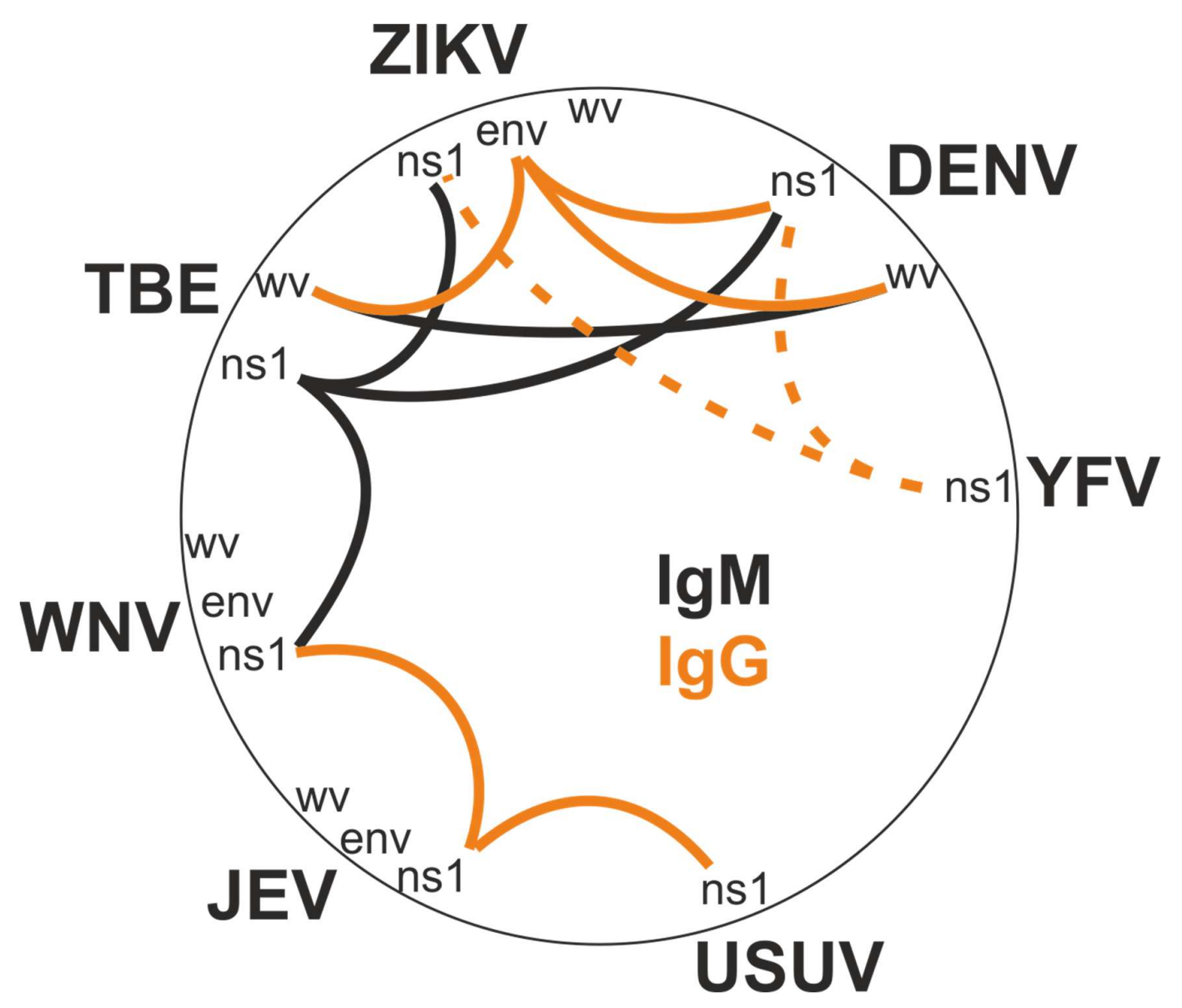

Figure 3. Overview of antigenic cross reactions ("Cross reactogram") encountered during development of a broad flaviserological multiplex assay [3]. Drawn by JB. 
10 of 13

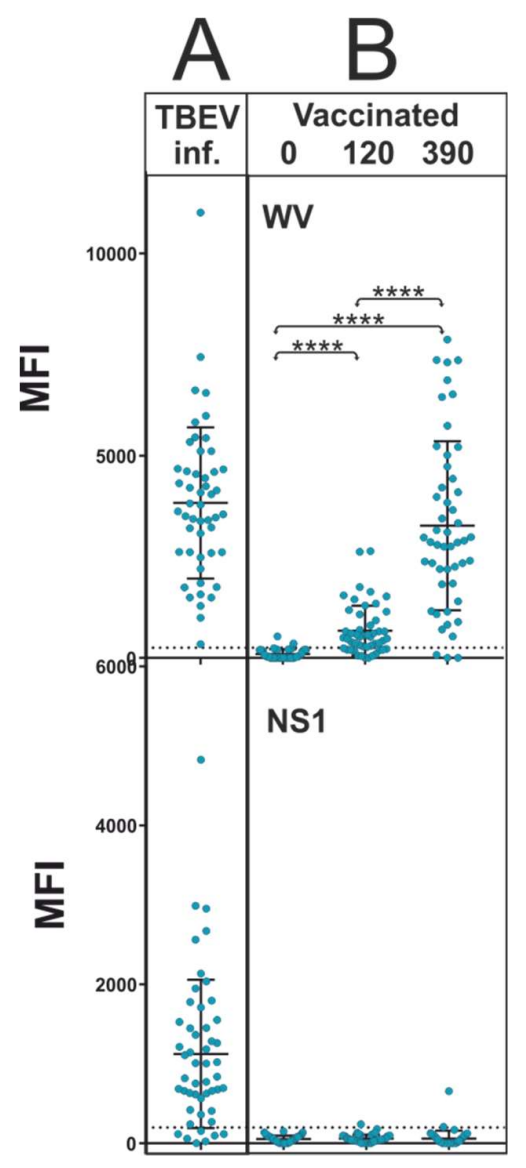

Figure 4. Distinction of IgG antibodies due to vaccination from those due to infection, amenable to high throughput population testing. Vaccinees do not develop antibodies to TBEV nonstructural protein NS1. Infected persons do so. Lane A: Sera from TBEV infected patients. Lane B: Sera from TBEV vaccinated persons. 0, 120 and 390 denote days of first, second and third vaccine dose, respectively. MFI: Median Fluorescent Intensity. From Euro Surveill. 2018;23(3):pii=17-00838. 


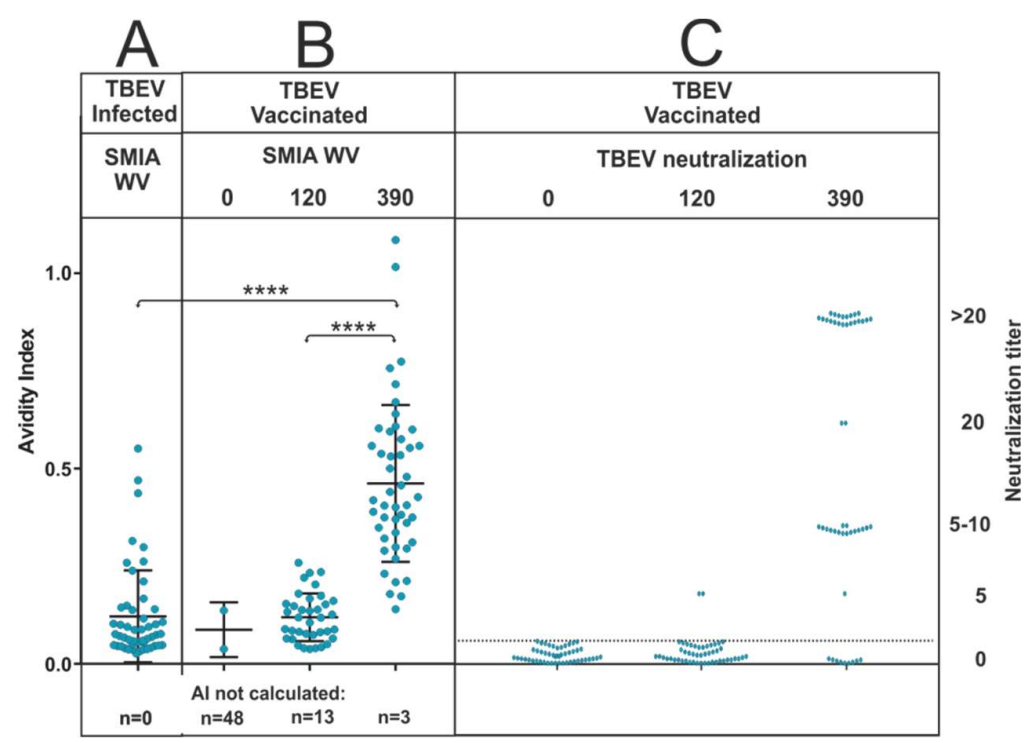

Figure 5. Neutralization titer ( C ) correlates with IgG avidity for TBEV antigen in SMIA. Column A shows results of sera from TBEV infected patients, B and C from TBEV vaccinated persons. WV: Whole virus antigen. 0, 120 and 390 denote days of first, second and third vaccine dose, respectively. Avidity index is the ratio of reactivity after and before urea treatment. From Euro Surveill. 2018;23(3):pii=17-00838.

Patents and conflicts of interest

JB submitted a patent on some of the described techniques [24].

\section{References}

1. Albinsson, B.; Vene, S.; Rombo, L.; Blomberg, J.; Lundkvist, A.; Ronnberg, B. Distinction between serological responses following tick-borne encephalitis virus (TBEV) infection vs vaccination, Sweden 2017. Euro Surveill 2018, 23, doi:10.2807/1560-7917.ES.2018.23.3.17-00838.

2. Rizwan, M.; Ronnberg, B.; Cistjakovs, M.; Lundkvist, A.; Pipkorn, R.; Blomberg, J. Serology in the Digital Age: Using Long Synthetic Peptides Created from Nucleic Acid Sequences as Antigens in Microarrays. Microarrays (Basel) 2016, 5, doi:10.3390/microarrays5030022.

3. Ronnberg, B.; Gustafsson, A.; Vapalahti, O.; Emmerich, P.; Lundkvist, A.; Schmidt-Chanasit, J.; Blomberg, J. Compensating for cross-reactions using avidity and computation in a suspension multiplex immunoassay for serotyping of Zika versus other flavivirus infections. Med Microbiol Immunol 2017, 206, 383-401, doi:10.1007/s00430-017-0517-y.

4. Ronnberg, B.; Vapalahti, O.; Goeijenbier, M.; Reusken, C.; Gustafsson, A.; Blomberg, J.; Lundkvist, A. Serogrouping and seroepidemiology of North European hantaviruses using a novel broadly targeted synthetic nucleoprotein antigen array. Infect Ecol Epidemiol 2017, 7, 1350086, doi:10.1080/20008686.2017.1350086.

5. Blomberg, J.; Blomberg, F.; Sjosten, A.; Sheikholvaezin, A.; Bolin-Wiener, A.; Elfaitouri, A.; Hessel, S.; Gottfries, C.G.; Zachrisson, O.; Ohrmalm, C., et al. No 
evidence for xenotropic murine leukemia-related virus infection in Sweden using internally controlled multiepitope suspension array serology. Clin Vaccine Immunol 2012, 19, 1399-1410, doi:10.1128/CVI.00391-12.

6. Blomberg, J.; Gottfries, C.G.; Elfaitouri, A.; Rizwan, M.; Rosen, A. Infection Elicited Autoimmunity and Myalgic Encephalomyelitis/Chronic Fatigue Syndrome: An Explanatory Model. Front Immunol 2018, 9, 229, doi:10.3389/fimmu.2018.00229.

7. Blomberg, J.; Sheikholvaezin, A.; Elfaitouri, A.; Blomberg, F.; Sjosten, A.; Mattson Ulfstedt, J.; Pipkorn, R.; Kallander, C.; Ohrmalm, C.; Sperber, G. Phylogeny-directed search for murine leukemia virus-like retroviruses in vertebrate genomes and in patients suffering from myalgic encephalomyelitis/chronic fatigue syndrome and prostate cancer. Adv Virol 2011, 2011, 341294, doi:10.1155/2011/341294.

8. Elfaitouri, A.; Berg, A.K.; Frisk, G.; Yin, H.; Tuvemo, T.; Blomberg, J. Recent enterovirus infection in type 1 diabetes: evidence with a novel IgM method. $J$ Med Virol 2007, 79, 1861-1867, doi:10.1002/jmv.21008.

9. Elfaitouri, A.; Hammarin, A.L.; Blomberg, J. Quantitative real-time PCR assay for detection of human polyomavirus infection. J Virol Methods 2006, 135, 207-213, doi:10.1016/j.jviromet.2006.03.006.

10. Elfaitouri, A.; Herrmann, B.; Bolin-Wiener, A.; Wang, Y.; Gottfries, C.G.; Zachrisson, O.; Pipkorn, R.; Ronnblom, L.; Blomberg, J. Epitopes of microbial and human heat shock protein 60 and their recognition in myalgic encephalomyelitis. PLoS One 2013, 8, e81155, doi:10.1371/journal.pone.0081155.

11. Elfaitouri, A.; Mohamed, N.; Fohlman, J.; Aspholm, R.; Frisk, G.; Friman, G.; Magnius, L.; Blomberg, J. Quantitative PCR-enhanced immunoassay for measurement of enteroviral immunoglobulin $\mathrm{M}$ antibody and diagnosis of aseptic meningitis. Clin Diagn Lab Immunol 2005, 12, 235-241, doi:10.1128/CDLI.12.2.235-241.2005.

12. Elfaitouri, A.; Shao, X.; Mattsson Ulfstedt, J.; Muradrasoli, S.; Bolin Wiener, A.; Golbob, S.; Ohrmalm, C.; Matousek, M.; Zachrisson, O.; Gottfries, C.G., et al. Murine gammaretrovirus group G3 was not found in Swedish patients with myalgic encephalomyelitis/chronic fatigue syndrome and fibromyalgia. PLoS One 2011, 6, e24602, doi:10.1371/journal.pone.0024602.

13. Mohamed, N.; Elfaitouri, A.; Fohlman, J.; Friman, G.; Blomberg, J. A sensitive and quantitative single-tube real-time reverse transcriptase-PCR for detection of enteroviral RNA. J Clin Virol 2004, 30, 150-156, doi:10.1016/j.jcv.2003.08.016.

14. Nilsson, A.L.; Vaziri-Sani, F.; Broberg, P.; Elfaitouri, A.; Pipkorn, R.; Blomberg, J.; Ivarsson, S.A.; Elding Larsson, H.; Lernmark, A. Serological evaluation of possible exposure to Ljungan virus and related parechovirus in autoimmune (type 1) diabetes in children. $J$ Med Virol 2015, 87, 1130-1140, doi:10.1002/jmv.24127.

15. Ohrmalm, C.; Jobs, M.; Eriksson, R.; Golbob, S.; Elfaitouri, A.; Benachenhou, F.; Stromme, M.; Blomberg, J. Hybridization properties of long nucleic acid probes for detection of variable target sequences, and development of a hybridization prediction algorithm. Nucleic Acids Res 2010, 38, e195, doi:10.1093/nar/gkq777. 
16. Wang, Y.; Hedman, L.; Perdomo, M.F.; Elfaitouri, A.; Bolin-Wiener, A.; Kumar, A.; Lappalainen, M.; Soderlund-Venermo, M.; Blomberg, J.; Hedman, K. Microsphere-based antibody assays for human parvovirus B19V, CMV and T. gondii. BMC Infect Dis 2016, 16, 8, doi:10.1186/s12879-015-1194-3.

17. Salmon, D.A.; Dudley, M.Z.; Glanz, J.M.; Omer, S.B. Vaccine hesitancy: Causes, consequences, and a call to action. Vaccine 2015, 33 Suppl 4, D66-71, doi:10.1016/j.vaccine.2015.09.035.

18. Anonym. Measles. Availabe online: https://ecdc.europa.eu/en/measles (accessed on

19. Anonym. Surveillance and disease data for mumps. Availabe online: https://ecdc.europa.eu/en/mumps/surveillance-and-disease-data (accessed on

20. viruses, I.c.f.t.o. Virus Taxonomy. Classification and Nomenclature of Viruses. Ninth ICTV report; International Union of Microbiological Societies, Virology Division: 2015.

21. Viruses, I.c.o.T.o. The ICTV Report. Virus Taxonomy: The Classification and Nomenclature of Viruses; ICTVonline, 2018.

22. Westman, G.; Blomberg, J.; Yun, Z.; Lannfelt, L.; Ingelsson, M.; Eriksson, B.M. Decreased HHV-6 IgG in Alzheimer's Disease. Front Neurol 2017, 8, 40, doi:10.3389/fneur.2017.00040.

23. Blomberg, J.; Pipkorn, R. Enterovirus peptides. EPO0546031A, 1990.

24. Blomberg, J. Multiplex Immunoassay with Enhanced Specificity for Improved Serodiagnosis of Infection with Cross-Reactive Microbes. December 12, 2017, 2017. 